\title{
Knowledge Representation for Potential Field of Study Recognition
}

\author{
Santy Arbi A.Mappe \\ School of Information Systems, Curtin Business \\ School, Curtin University \\ Perth, Australia \\ e-mail: santylpmp@yahoo.com
}

\author{
Pornpit Wongthongtham \\ School of Information Systems, Curtin Business \\ School, Curtin University \\ Perth, Australia \\ e-mail: Ponnie.Clark@curtin.edu.au
}

\begin{abstract}
Knowledge Representation is a part of Artificial Intelligence that focuses on the formalism design. The knowledge about a specific domain is expressed epistemologically and computationally. One of the main reasons for this is that knowledge must be represented so as to easily identify the structure and characteristics of classes and the relationship among them. This paper will focus on the systematic investigation of ontology's formula that is presented by Description logics. We believe that Description logics be able to sketch, define, integrate and maintain the ontology.
\end{abstract}

Keywords - ontology; field of study; knowledge representation; description logic, artificial intelligence.

\section{INTRODUCTION}

Ontology is becoming important as a semantic foundation since it caters for the growing technologies[1]. Ontology plays an important role in describing the specification of conceptualization which can be used to represent the knowledge about specific domains and their relationships [2]. As well-defined by Gruber, "Ontology is a formal, explicit specification of shared conceptualization $^{\text {ee }}$ [3]. The purpose of ontology is to study of the categories of things in various domains [4]. One of the explanations of ontology is: an ontology is a vocabulary of interrelated terms which impose a structure on the domain and constrain the possible interpretation of terms [5]. Ontology offers the basis for modeling a subject domain [6] and it has become an extensive area of research that merits specific analysis. Ontology can offer solutions to educational problems by representing hierarchical and navigational relationships to establish a shared understanding of a specific domain [7]. We have previously developed an Ontological Framework for Field of Study Recognition in education in order to help schools, parents and students to improve students' performance by determining and taking into account the potential talent and interest of students. The framework has three stages beginning with the development of a basic ontology for domain representation. In order to assist students to decide on an appropriate field of study, a standard to represent knowledge as a set of concepts is needed [8]. Two key factors that must be taken into account in this approach are personalities and field of study. These two domains are presented formally in order to avoid misunderstanding and/or misinterpretation[8]. By explicitly defining the concepts, it will be possible to obtain valuable knowledge about the fields of study and students' personalities. In order to demonstrate ontologybased user modeling, both ontologies will be presented using Description Logic.

\section{RELATED WORK}

Various research projects on ontology representation have been conducted and discussed by researchers [9]. In particular, regarding education, Dicheva [7] stated: "the field of applying ontological research in education is fairly young it is already quite broad and fuzzy". Researchers have used various means to represent and use cutting-edge technology in order to enhance ontology representation. Ontologies are being used in more and more areas to capture and formalize domain knowledge [10].

\section{A. Ontology in Education}

To date, a substantial amount of work has been undertaken on ontology representation in the area of education; however, no-one has discussed or developed an ontology for both talent and interest in order to determine the appropriate field of study for a prospective student. Some of them focus on course content while others discuss ELearning.

Boyce has developed an ontology for the educational context, particularly to support the content and instructional design [11]. Borges also focused on the modeling of educational content [12]. Fig. 1 shows example tree of Boyce's ontology. Dicheva [13] developed the ontology for an educational portal and represented the ontology as a topic map. Another project, called OMNIBUS, is an ontology designed for understanding learning, instruction and instructional design. 


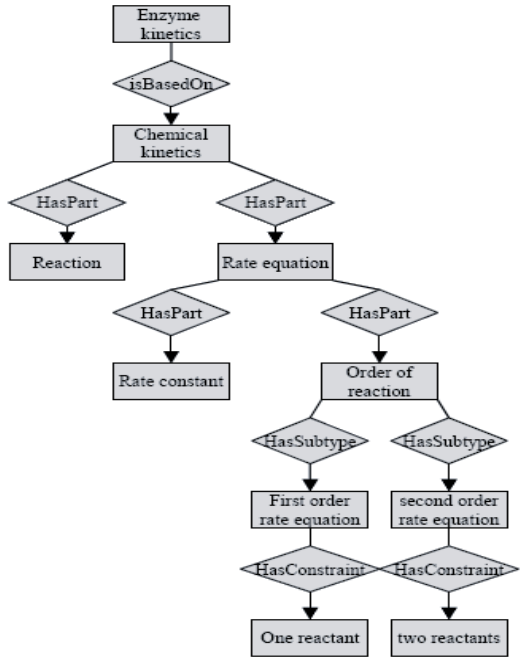

Fig. 1. Example tree of Boyce's ontology

\section{B. Description Logic in Education Domain Representation.}

Ontology is a conceptual model in a domain which is used to represent the concepts and relationship through them [3], which contains a description of the specific domain. It describes the semantics in multiple different aspects and dimensions of the content to be able to cover the semantic needs [14]. Ontology was evolved as a cognitive tool which is broadly used to produce concept maps, mind maps and other visual representations for learning purposes[13].

Description Logics (DLs) are used widely in the education environment, in particular to represent the reused parts of the knowledge. Nenad explains that description logics reasoning techniques are more useful than case-based reasoning[15].

Krdzavac, in her research using DLs reasoning techniques in the web-based education environment, explains how description logics reasoning are used for an intelligent analysis of student solutions. The findings of this project are useful for the education field but it focused on learning and teaching materials, not on the discovery of students' talents and interests,

All the researches have contributed something of value to education. However, in terms of assisting students to choose an appropriate field of study, little of the research can be used since the ontology developed to date does not support the aim of this research.

\section{III.ONTOLOGY DESIGN IN THEDOMAIN OF FIELD OF STUDY RECOGNITION}

Previous research regarding the identification of the right field of study for students based on talent and interest was done by Mappe et al. [8]. They mentioned that there are two factors required to discover the appropriate field of study i.e. field of study and personalities.

Personalities ontology consists of the physical characteristics that indicate talent such as fingerprint pattern, blood group type and the form of handwriting. The other content of personalities is interest that represents the attention given to the specific area of study.

\section{IV.ONTOLOGY REPRESENTATION FOR FIELD OF STUDY RECOGNITION}

Gruber originally defined the notion of an ontology as an "explicit specification of a conceptualization"[3].Ontology is used to support interoperability and common understanding [16]. In this paper, we aim to have an ontology to represent the prospective features of students by incorporating the characteristics of human called talent and the personal attention on particular field of study namely interest. An ontology allows us to specify, in a meaningful and open way, not only the concepts but also the relationships in a domain of interest [17]. As explained above, two ontologies will be defined here: personalities ontology (the talent) and field of study ontology (the interest)[8].

Ontology can be represented in various formats to understand the domain and it allows knowledge to be exchanged among applications. The appropriate ontology representation language becomes a significant issue in designing the ontology [18]. Various popular methods have been developed to express the ontology and knowledge representation such as Description Logics (DLs), Semantic Networks and Unified Modeling Language (UML). However, in this paper, DLs will be chosen as the alternative formalisms for representing the ontologies.

\section{A. Personalities Ontology}

In this part, Ontology Personalities will be represented. Several key criteria are applied when determining giftedness [8]. Ability is not the only factor used to determine talent; the personalities in his/her daily activities must also be considered[19]. Three personal characteristics of talent will be taken into account: blood group, fingerprint pattern and handwriting pattern.

The concept above will be presented in Ontology format, and DLs are used to construct the knowledge modeling and accurately describe the objects with parts connected in arbitrary ways.

The steps used to formally represent the knowledge of domain in DLs include: defining the concepts, and specifying the properties of objects and individuals in the domain.

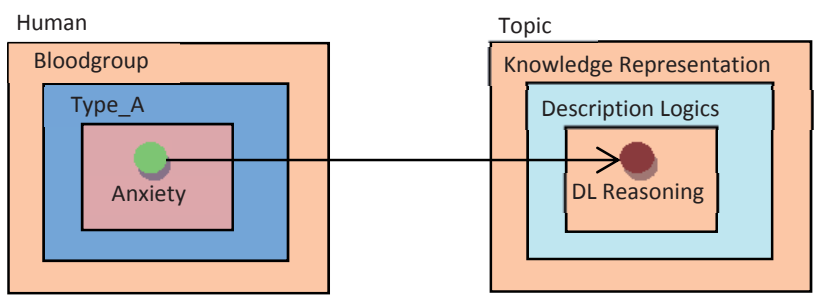

Fig. 2. An example of Personalities ontology

Simply, the personalities ontology can be depicted as shown in Fig 2. Human has Talent and Interest. Talent 
consists of 3 parts: blood group, fingerprint and graphology, while Interest consists of the collection of areas of study.

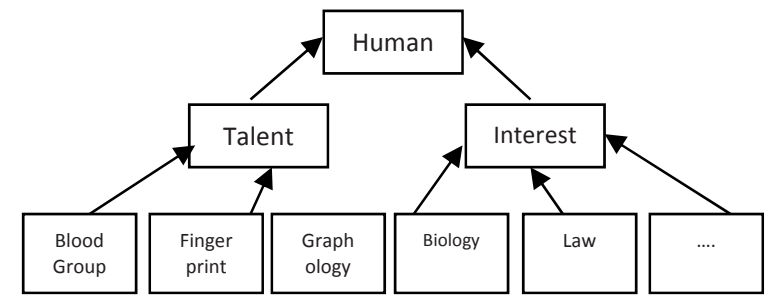

Fig. 3. Hierarchy of Human Personalities

The basic entities for representing entities using DLs called concepts which correspond to formula in mathematics logic. Generally, concepts are built from concept names, role names and constructors. Fig. 3 clearly shows that human personalities consist of talent and interest. Talent consists of blood group, fingerprint and graphology, and the other side, interest comprised of the possibilities of field of study. Furthermore, the hierarchy can be described in DLs format. For example, "All Human has Talent some Talent and has Interest some Interest". This concept can be expressed as follows:

\section{Human $\sqsubseteq \exists$ hasTalent.Talent $\sqcap$ $\exists$ hasInterest.Interest}

In RDF syntax, the concept can be shown as:

$<$ owl:Classrdf:about="\#Human"/>

$<$ owl:Classrdf:about="\#Interest">

$<$ rdfs:subClassOfrdf:resource="\#Human"/>

$<$ owl:Class $>$

$<$ owl:Classrdf:about="\#Talent" $>$

$<$ rdfs:subClassOfrdf:resource="\#Human"/>

$<$ owl:Class $>$

$</$ owl:Class $>$

Assume that we want to define the concept of Human as having at least one blood group which corresponds to a number of characteristics. Using description logics concepts, it can be seen as:

\section{Human $\sqsubseteq \exists$ hasTalent.Talent $\sqcap$ Talent $\exists \forall$}

BloodGrouphasCharacteristics.Characters

The formula can be presented in the RDF syntax as follows:

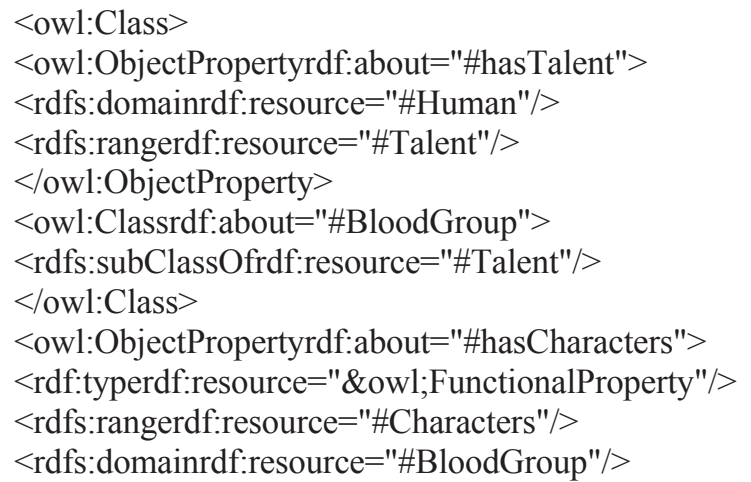

$<$ owl:Class $>$

$<$ rdfs:domainrdf:resource="\#Human"/>

$<$ rdfs:rangerdf:resource $=" \#$ Talent" $/>$

$<$ owl:ObjectProperty $>$

$<$ owl:Classrdf:about="\#BloodGroup">

$<$ rdfs:subClassOfrdf:resource="\#Talent" $>$

<rdf:typerdf:resource="\&owl;FunctionalProperty"/>

$<$ rdfs:domainrdf:resource="\#BloodGroup"/>

$</$ owl:ObjectProperty $>$

$</$ owl:Class $>$

\section{B. Field of Study Ontology Representation}

Murphy [20] declares that students should have a clear perspective of their studies. This perspective should be a succinct statement and present a definite sense of what $\mathrm{s} /$ he wants to do together with a passion for the specific field of study.

Interest, personality and talent must be considered when selecting a major[21]. Gagne believes that giftedness is a natural ability expressed by the individual in a particular domain(s) such as intellectual, creative, socio affective and sensorimotor[21].

Interest is the attention given to a specific field of study. In this session, we focused on developing the field of study ontology based on the senior high school curriculum in Indonesia.

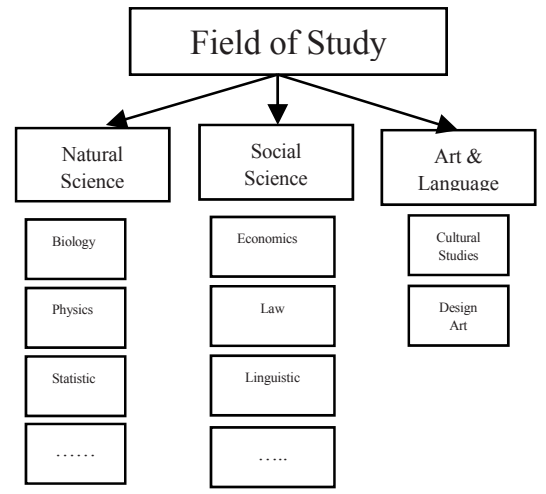

Fig. 4. Curriculum Hierarchy

From Fig. 4, we can see that the Field of Study consists of three parts: Natural Science, Social Science and Art \& Language, each part of which is correlated to characters.

For example, if we want to show that Biology is a part of Natural Science, in DLs the concept can be described as:

NaturalScience $\sqsubseteq \exists$ hasPart.Biology

Every part of Natural Science is correlated to characters.

NaturalScience巨iscorrelatedto.Characters

In RDF syntax, the concept would be written as:

$<$ owl:Classrdf:about="\#NaturalScience" $>$

$<$ rdfs:subClassOfrdf:resource="\#FieldOfStudy"/>

$</$ owl:Class $>$

$<$ owl:Classrdf:about="\#FieldOfStudy"/>

$<$ owl:Classrdf:about="\#Characters"/>

$<$ owl:Classrdf:about="\#Biology">

$<$ rdfs:subClassOfrdf:resource="\#NaturalScience"/>

$<$ owl:Class $>$

<owl:ObjectPropertyrdf:about="\#haspart">

$<$ rdf:typerdf:resource="\&owl;FunctionalProperty"/>

$<$ rdfs:rangerdf:resource="\#Biology" $>$ 


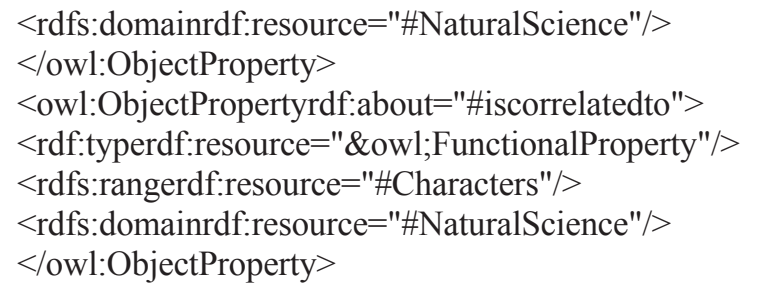

\section{V.CONCLUSION}

This paper focused on the development of two ontologies: i) personalities ontology, and ii) field of study ontology. In future work, we intend to develop the matching engine and selection engine in Java to match both ontologies. It is anticipated that this work will be useful in the future, not only for students, parents and teachers to select the appropriate field of study related to talent and interests in order to support future individual directions, but also it assists government bodies to decide on and allocate resources in a timely fashion.

\section{REFERENCES}

[1] McGuinness, D. Ontologies Come of Age The Semantic Web: Why, What, and How., 2001.

[2] Noy, N.F. and C.D. Hafner, The State of the Art in Ontology Design : A Survey and Comparative Review, in AI Magazine.

[3] Gruber, T.R., A Translation Approach to Portable Ontology., in Technical Report KSL 92-71, Knowledge Acquition1993, Stanford University: California. p. 199-220.

[4] Sowa, J.F., Knowledge Representation: Logical, Philosophical, and Computational Foundations, 2000, Brooks/Cole, Thomson Learning.

[5] Uschold, M., Knowledge Level Modelling: Concepts and Terminology. The Knowledge Engineering Review, 1998. 13: p. N1.

[6] Kalinichenko, L. and M. M, Ontological Modeling. Proceedings of the 5 th RussianConferenceon Digital LibrariesRCDL2003, 2003.

[7] Dicheva, D., et al., Ontological Web Portal for Educational Ontologies, in 12th International Conference on Artificial Intelligence in Education (AIED'05)2005, SW-EL'05: Applications of Semantic Web Technologies for E-Learning: Amsterdam. p. 19.

[8] Mappe, S.A.A., P. Wongthongtham, and O. Hussain, An Ontological Framework for Field of Study Recognition in Education. Proceedings of 5th International Conference in Human System Interaction (HSI 2012), IEEE, Perth, Australia, 2012.

[9] Shapiro, S., Hermeneutics, Knowledge Acquisition, Knowledge Representation, in Encyclopedia of Artificial Intelligence1992, John Wiley \& Sons, Inc. p. 596-611, 719-742, 743-758.

[10] Uschold, M. and M. Gruniger, Onthologies: Principles, Methods and Applications. Knowledge Engineering Review 1996. 11 Number 2.

[11] Boyce, S. and C. Pahl, Developing Domain Ontologies for Course Content. Educational Technology \& Society, 2007. 10(3): p. 13.

[12] Borges, V.A. and E.F. Barbosa, Using Ontologies for Modeling Educational Content. SEWL 2009, 2009.

[13] Dicheva, D., Ontologies and Semantic Web for E-Learning, in Handbook on Information Technologies For Education and Training, H.H. Adelsberger, et al., Editors. 2008, Springer: Verlag Heidelberg. p. 47-65.

[14] Jokela, S., M. Turpeinen, and R. Sulonen. Ontology Development for Flexible Content. in Ontology Development for Flexible Content. 2000. Hawaii.

[15] Cardoso, J., The Semantic Web Vision: Where are We? IEEE Intelligent Systems, September, 2007: p. 5.

[16] Dou, D., D. McDermott, and P. Qi, Ontology Translation on the Semantic Web. Journal on Data Semantic II, 2004: p. 28.

[17] Cordeiro, J., B. Antunes, and P. Gomes. Context-based recommendation to support problem solving in software development. in Recommendation Systems for Software Engineering (RSSE), 2012 Third International Workshop on. 2012.

[18] Cranefield, S., S. Haustein, and M. Purvis, UML-Based Ontology Modelling for Software Agents.

[19] Tannenbaum, A.J., Gifted children: Psychological and educational perspectives. 1983.

[20] Malik, S.K., N. Prakash, and S.A.M. Rizvi, Developing an University Ontology in Education Domain using Protégé for Semantic Web. International Journal of Engineering Science and Technology, 2010. 2(9): p. 9.

[21] Wang, X. and C.W. Chan, Ontology Modeling Using UML, Departement of Computer Science, University of Regina: Canada. 